\title{
Numerical solution of free-boundary problems in fluid mechanics. Part 1. The finite-difference technique
}

\author{
By G. RYSKIN† AND L. G. LEAL \\ Department of Chemical Engineering, California Institute of Technology, \\ Pasadena, California 91125
}

(Received 11 April 1983 and in revised form 27 April 1984)

\begin{abstract}
We present here a brief description of a numerical technique suitable for solving axisymmetric (or two-dimensional) free-boundary problems of fluid mechanics. The technique is based on a finite-difference solution of the equations of motion on an orthogonal curvilinear coordinate system, which is also constructed numerically and always adjusted so as to fit the current boundary shape. The overall solution is achieved via a global iterative process, with the condition of balance between total normal stress and the capillary pressure at the free boundary being used to drive the boundary shape to its ultimate equilibrium position.
\end{abstract}

\section{Introduction}

We are concerned in this paper, and the two papers that follow, with some specific examples of the class of so-called 'free-boundary' problems of fluid mechanics. This class of problems is characterized by the existence of one boundary (or more) of the flow domain whose shape is dependent upon the viscous and pressure forces generated by the fluid motion. In this case, the shape of the boundary and the form of the velocity and pressure fields in the fluid are intimately connected, and one must solve for the boundary shape as a part of the overall solution of a particular problem. The most common problems of this type in fluid mechanics occur in the motions of two immiscible fluids which are contiguous at a common interface. In Parts 2 and 3 of this series (Ryskin \& Leal 1984a,b), we discuss numerical results for two specific problems involving the motion of a bubble in a viscous incompressible Newtonian fluid; namely buoyancy-driven motion through an unbounded quiescent fluid, and motion in an axisymmetric straining flow. In the present paper, we discuss a general numerical solution scheme, used in Parts 2 and 3 , which would be expected to carry over to the solution of other free-boundary problems that involve a gas-liquid interface.

The existing published literature on free-boundary problems in fluid mechanics is quite extensive in number, but limited in scope. Three distinct solution methods can be identified. By far the majority of papers are concerned with asymptotic or limiting cases in which the interface shape, while unknown, deviates only slightly from some predefined configuration. In the case of bubble motions, for example, a number of authors have used the so-called 'domain perturbation' method to solve for the first (infinitesimal) deviations from a spherical bubble in a variety of flows (ef. Taylor 1934; Taylor \& Acrivos 1964). In addition, a similar approach has been used to consider

$\dagger$ Present address: Department of Chemical Engineering, Northwestern University, Evanston, Illinois 60201. 
the first deviations from the limiting form of a slender body with an arbitrarily small radius-to-length ratio, which is relevant, for example, to low-viscosity drops in uniaxial extensional flows with a sufficiently high strain rate (Taylor 1964; Acrivos \& Lo 1978). A second method of solution suitable for free-boundary problems is the so-called boundary-integral technique, which is restricted to the limiting cases of either zero Reynolds number, where the governing differential equations are the linear Stokes equations, or inviscid, irrotational flow, where the governing differential equations reduce to Laplace's equation. In this method, fundamental solutions of the linear governing equations are used to reduce the general $n$-dimensional problem to the solution of a set of $(n-1)$-dimensional integral equations. The boundary-integral method is not restricted to small deformations. Indeed, solutions have been obtained which exhibit large departures from a predefined shape (Youngren \& Acrivos 1976; Miksis, Vanden-Broeck \& Keller 1981; Lee \& Leal 1982). However, the restriction to creeping or potential flows reduces its usefulness. The third and most important class of free-boundary problems is that in which neither of the restrictions of small deformation nor linear governing differential equations is present. This is, quite simply, the general problem at finite Reynolds number, which clearly requires a fully numerical method of solution. This case has received relatively little attention to date. Most of the solutions which have been obtained were developed using a finite-element formulation of the numerical problem. Here we consider an alternative approach based upon a finite-difference approximation of the governing equations.

The finite-difference method that we have developed incorporates a numerically generated orthogonal coordinate system, which is 'boundary-fitted' in the sense that all boundary surfaces of the solution domain (including the free boundary whose shape is determined as part of the solution) coincide with a coordinate line (or surface) of the coordinate system. Thus the problem of interpolation between nodal points of the finite-difference grid when the latter is not coincident with physical boundaries is avoided altogether. Indeed, the existence of the interpolation problem in the first place is seen to be a consequence of the use of the common, analytically generated coordinate systems, such as cylindrical, spherical, etc., when the latter do not correspond to the natural boundaries of the solution domain.

A full description of the procedure for generation of an orthogonal boundary-fitted coordinate system has already been given in Ryskin \& Leal (1983, hereinafter called I). The present paper will focus on implementation of this procedure in a full numerical algorithm for fluid-mechanics problems in which the free boundary is a gas-liquid interface. The mapping procedure is presently restricted to two-dimensional and axisymmetric flow domains. For the problems currently under investigation, we additionally restrict ourselves to steady motions. The shape of the free boundary is determined via an iterative procedure, with the coordinate system changed at each step to match the current approximation to the free-boundary shape.

\section{Problem formulation}

In this section we outline the mathematical formulation of a typical free-boundary problem in which the free boundary is a gas-liquid interface that is assumed to be completely characterized by a constant (i.e. spatially uniform) surface tension. In effect, we are assuming that the interface is free of surfactant and the system is isothermal. We assume that the boundary geometry and flow fields are both axisymmetric and steady. The steady-state assumption can be relaxed, in principle, by suitable modification of the method and equations of this paper. The assumption of axisymmetry is required by the mapping algorithm in its present form, and is also 
necessary in order to keep the computing cost within reasonable limits. We assume that the liquid in our system is incompressible and Newtonian, and that its density and viscosity are sufficiently large compared with those of the gas that the dynamic pressure and stress fields in the gas at the interface can be neglected compared to those on the liquid side.

We denote the 'boundary-fitted' coordinate system as $(\xi, \eta, \phi)$, with $\phi$ the azimuthal angle measured about the axis of symmetry. In view of the assumed axisymmetry, these boundary-fitted coordinates can be connected with the common cylindrical coordinates $(x, \sigma, \phi)$ (with the axis of symmetry being the $x$-axis) via a pair of mapping functions $x(\xi, \eta)$ and $\sigma(\xi, \eta)$, which satisfy the covariant Laplace equations (see I)

$$
\left.\begin{array}{c}
\frac{\partial}{\partial \xi}\left(f \frac{\partial x}{\partial \xi}\right)+\frac{\partial}{\partial \eta}\left(\frac{1}{f} \frac{\partial x}{\partial \eta}\right)=0, \\
\frac{\partial}{\partial \xi}\left(f \frac{\partial \sigma}{\partial \xi}\right)+\frac{\partial}{\partial \eta}\left(\frac{1}{f} \frac{\partial \sigma}{\partial \eta}\right)=0 .
\end{array}\right\}
$$

Here the function $f(\xi, \eta)$ is the so-called 'distortion function' representing the ratio $h_{\eta} / h_{\xi}$ of scale factors $\left(h_{\eta} \equiv\left(g_{\eta \eta}\right)^{\frac{1}{2}}, h_{\xi} \equiv\left(g_{\xi \xi}\right)^{\frac{1}{2}}\right)$ for the boundary-fitted coordinate system. In the 'strong-constraint' method developed in I for free-boundary problems, the distortion function can be freely specified to provide control over the density of coordinate lines in the boundary-fitted system. With respect to the $(\xi, \eta, \phi)$-system, the mapping is always defined in such a way that the solution domain (for any arbitrary fixed $\phi$ ) is the unit square

$$
0 \leqslant \xi, \eta \leqslant 1 .
$$

Boundary conditions for the mapping functions $x(\xi, \eta)$ and $\sigma(\xi, \eta)$ were described in detail in I. In $\$ 4$ we focus on boundary conditions at the free surface, and the corresponding numerical method of adjusting the interface shape at each step in an iterative solution scheme, with the shape change based upon the imbalance of normal stress and surface-tension forces calculated from a previous guess of the interface shape.

The fluid-mechanics part of the problem, then, is to obtain solutions of the Navier-Stokes equations using a finite-difference approximation in the boundaryfitted $(\xi, \eta)$-coordinates. With axisymmetry assumed, the Navier-Stokes equations are most conveniently expressed in terms of the stream function $\psi$ and vorticity $\omega$ in the form

where

$$
\begin{gathered}
L^{2}(\omega \sigma)-\frac{R}{2} \frac{1}{h_{\eta} h_{\xi}}\left[\frac{\partial \psi}{\partial \xi} \frac{\partial}{\partial \eta}\left(\frac{\omega}{\sigma}\right)-\frac{\partial \psi}{\partial \eta} \frac{\partial}{\partial \xi}\left(\frac{\omega}{\sigma}\right)\right]=0, \\
L^{2} \psi+\omega=0,
\end{gathered}
$$

and $R$ is an appropriate Reynolds number for the specific problem of interest. In terms of the mapping functions $x(\xi, \eta)$ and $\sigma(\xi, \eta)$, the scale factors that appear in these equations are

$$
\begin{aligned}
& h_{\xi}=\left\{\left(\frac{\partial x}{\partial \xi}\right)^{2}+\left(\frac{\partial \sigma}{\partial \xi}\right)^{2}\right\}^{\frac{1}{2}}, \\
& h_{\eta}=\left\{\left(\frac{\partial x}{\partial \eta}\right)^{2}+\left(\frac{\partial \sigma}{\partial \eta}\right)^{2}\right\}^{\frac{1}{2}} \cdot
\end{aligned}
$$


We assume, for convenience, that the coordinate mapping is defined with $\xi=1$ corresponding to the interface, and $\eta=0$ and 1 to the symmetry axes. Then boundary conditions at the symmetry axes are

$$
\psi=0, \quad \omega=0 \text { at } \eta=0,1 .
$$

At the gas-liquid interface $(\xi=1)$ we require

$$
\psi=0,
$$

corresponding to zero normal velocity in the assumed steady-state solution;

$$
\omega-2 \kappa_{(\eta)} u_{\eta}=0
$$

corresponding to the condition of zero tangential stress (where $\kappa_{(\eta)}$ is the normal curvature of the interface in the $\eta$-direction and $u_{\eta}$ is the tangential velocity); and

$$
\tau_{\xi \xi}-\frac{4}{W}\left(\kappa_{(\eta)}+\kappa_{(\phi)}\right)=0
$$

representing the balance between the normal-stress contributions due to pressure and viscous forces on the one hand and the capillary force on the other. In (9) $\kappa_{(\phi)}$ is the normal curvature in the $\phi$-direction, $W$ is a (dimensionless) Weber number measuring the ratio of characteristic pressures due to inertial and capillary forces at the interface, and $\tau_{\xi \xi}$ is the total normal stress, which includes both static and dynamic pressure and viscous contributions. In terms of $(\xi, \eta, \phi)$-coordinates, $\tau_{\xi \xi}$ can be calculated in the form

$$
\tau_{\xi \xi}=-p+\frac{8}{R} e_{\xi \xi}=-p-\frac{8}{R} \frac{1}{\sigma h_{\eta}} \frac{\partial}{\partial \eta}\left(\sigma u_{\eta}\right)
$$

To obtain the pressure at the interface, we use the equation of motion including all body-force terms, since these contribute to the hydrostatic term in $p$. Expressions for the normal curvatures $\kappa_{(\eta)}$ and $\kappa_{(\phi)}$ are obtained easily in terms of the so-called connection coefficients of the $(\xi, \eta, \phi)$-coordinates, as shown in I. In particular,

$$
\left.\begin{array}{l}
\kappa_{(\eta)}=-\frac{1}{h_{\eta} h_{\xi}} \frac{\partial h_{\eta}}{\partial \xi}, \\
\kappa_{(\phi)}=-\frac{1}{\sigma h_{\xi}} \frac{\partial \sigma}{\partial \xi} .
\end{array}\right\}
$$

However, from a computational point of view, it is more convenient to differentiate parallel to the interface rather than normal to it, and to avoid differentiation of a scale factor. Thus, using the general properties of an orthogonal mapping (see I),

$$
\begin{gathered}
\kappa_{(\eta)}=\frac{1}{h_{\eta}^{3}}\left(\frac{\partial x}{\partial \eta} \frac{\partial^{2} \sigma}{\partial \eta^{2}}-\frac{\partial^{2} x}{\partial \eta^{2}} \frac{\partial \sigma}{\partial \eta}\right), \\
\kappa_{(\phi)}=-\frac{1}{\sigma h_{\eta}} \frac{\partial x}{\partial \eta} .
\end{gathered}
$$

The boundary conditions at $\xi=0$ (the far-field boundary in many cases) depend on the particular problem. If the flow domain is two-dimensional rather than axisymmetric, suitable modifications of (2)-(9) are made easily, but will not be pursued here.

It may be noted that the complete stream-function and vorticity fields can be determined for an axisymmetric interface of specified shape using only conditions (7) 
and (8). The normal-stress balance (9) then provides a consistency condition on the interface shape, as well as a basis for determining an improved estimate of that shape when the condition is not satisfied.

\section{Solution algorithm}

The problem, from a numerical point of view, is to solve simultaneously the differential equations (1), (2) and (3) for the mapping functions and the streamfunction/vorticity fields subject to conditions (7)-(9) at the interface $\xi=1$, plus appropriate additional boundary conditions at the other boundaries $\xi=0$ and $\eta=0,1$ as indicated above.

A simple approach which we have found to be efficient and stable for solution of this coupled fluid-dynamics-mapping problem involves an iterative procedure that can be summarized broadly as follows:

1. Choose some initial shape of the gas-liquid interface together with the corresponding orthogonal coordinate system (usually taken from a known solution or obtained analytically, as for a spherical bubble).

2. Obtain an approximation to the stream-function and vorticity fields by carrying out some small number of iterations on (2) and (3), subject to the boundary conditions (6)-(8), plus the appropriate conditions at $\xi=0$. In practice, we have found that the most rapid convergence of the overall solution algorithm is usually attained by a single iteration at this step.

3. Check the normal-stress condition (9), and if it is not satisfied, modify the interface shape so as to reduce the imbalance between the total stress $\tau_{\xi \xi}$ and the surface-tension term $4\left(\kappa_{(\eta)}+\kappa_{(\phi)}\right) / W$, and compute a new (approximate) boundaryfitted coordinate system by carrying out some number of iterations (usually one) on (1), plus boundary conditions.

4. Return to step 2 and repeat until all equations and boundary conditions are satisfied.

In the subsequent sections of this paper we consider the details of this numerical algorithm. In particular, we discuss implementation of the tangential-stress condition (8), the ADI technique used to solve the differential equations (1), plus (2) and (3), and finally the method of modifying the interface shape in accord with (9).

\section{Details of the solution algorithm}

\subsection{Discretization and solution by $A D I$}

At each step of the iterative solution algorithm described above, a new estimate of the interface shape is obtained and both the equations (1) defining the coordinate mapping and the equations of motion (2) and (3) must be solved at least approximately. Although a variety of finite-difference schemes exist which could be used for solution of these equations, it is not possible to compare them exhaustively to ensure an optimal approach for each particular problem, and the choice of method must ultimately be made by intuition guided by past experience. The present work uses the familiar ADI scheme of Peaceman and Rachford (see e.g. Richtmyer \& Morton 1967), both for (1) and the equations of motion (2) and (3). This choice is predicated primarily on the observation, which is confirmed by numerical experimentation, that it would be inefficient to try to find highly accurate solutions either for the stream-function/vorticity fields or the coordinate transformation at each intermediate step of the global iteration when the boundary shape is not close to its final converged 
result. This consideration rules out non-iterative procedures for the solution of (1)-(3); the popular multigrid approach also becomes less attractive, since one would have to perform the full multigrid cycle (i.e. fine to coarse grid and back) at each global iteration in order to calculate a reasonable approximation to the quantities entering the normal stress imbalance at this iteration (see step 3 in the above algorithm). In effect, our global iterative algorithm is equivalent to solving an artificial (non-physical) time-dependent problem, and for time-dependent problems on a rectangle the advantages of multigrid over such methods as ADI have not been demonstrated clearly.

In order to illustrate the ADI procedure in our present context, it is convenient to express (1)-(3) in a standard form

$$
0=f^{2} \frac{\partial^{2} w}{\partial \xi^{2}}+\frac{\partial^{2} w}{\partial \eta^{2}}+q_{1} \frac{\partial w}{\partial \xi}+q_{2} \frac{\partial w}{\partial \eta}+q_{3} w+q_{4}
$$

in which the $q$ 's are coefficients that do not depend explicitly on $w$. The ADI procedure is then implemented by imbedding (13) in a fictitious time-dependent problem of parabolic type, and discretizing in such a way that derivatives in the spatial directions are alternatively treated implicitly at one half-step in 'time' and explicitly at the next half-step. Denoting the value of an independent variable at each full iteration $n$ by a superscript, and the intermediate (half-step) value by $\sim$, we have

$$
\begin{gathered}
\frac{\tilde{w}-w^{n}}{\frac{1}{2} \Delta t}=f^{2} \frac{\delta^{2} \tilde{w}}{\delta \xi^{2}}+\frac{\delta^{2} w^{n}}{\delta \eta^{2}}+q_{1}^{n} \frac{\delta \tilde{w}}{\delta \xi}+q_{2}^{n} \frac{\delta w^{n}}{\delta \eta}+\left(\frac{q_{3}^{n}-\left|q_{3}^{n}\right|}{2}\right) \frac{\tilde{w}+w^{n}}{2} \\
+\left(\frac{q_{3}^{n}+\left|q_{3}^{n}\right|}{2}\right) w^{n}+q_{4}^{n}, \\
\begin{array}{r}
\frac{w^{n+1}-\tilde{w}}{\frac{1}{2} \Delta t}=f^{2} \frac{\delta^{2} \tilde{w}}{\delta \xi^{2}}+\frac{\delta^{2} w^{n+1}}{\delta \eta^{2}}+q_{1}^{n} \frac{\delta \tilde{w}}{\delta \xi}+q_{2}^{n} \frac{\delta w^{n+1}}{\delta \eta}+\left(\frac{q_{3}^{n}-\left|q_{3}^{n}\right|}{2}\right) \frac{\tilde{w}+w^{n+1}}{2} \\
+\left(\frac{q_{3}^{n}+\left|q_{3}^{n}\right|}{2}\right) w^{n}+q_{4}^{n} .
\end{array}
\end{gathered}
$$

We utilize a uniform finite-difference grid in the $(\xi, \eta)$-coordinates, with control over mesh density affected by the form chosen for the distortion function $f(\xi, \eta)$. The symbols $\delta / \delta \xi$ or $\delta / \delta \eta$ are used in $(14 a, b)$ to denote centred-difference approximations of the indicated partial derivatives. The resulting systems of linear algebraic equations along each coordinate line are tridiagonal and can be solved very efficiently.

The only unusual feature of the discretization in $(14 a, b)$ is that involving the term $q_{3} w$ in (13). The 'obvious' form $q_{3}^{n}\left(\tilde{w}+w^{n}\right) / 2$ for the first half-step and $q_{3}^{n}\left(\tilde{w}+w^{n+1}\right) / 2$ for the second was found to lead to instability in the solution of the vorticity equation (2) for $R>O(50)$, even with frozen $\psi$ and boundary conditions. This instability was, at first, thought to be due to the use of centred-difference approximations in the $q_{1}$ and $q_{2}$ terms. However, putting $q_{1}$ and $q_{2}$ identically equal to zero (for testing purposes) did not prevent instability, while $q_{3}=0$ did. It is evident that a differential equation of the form

$$
\frac{\partial w}{\partial t}=q_{3} w
$$

will exhibit exponential growth of $w$ if $q_{3}$ is positive. Such growth is, of course, restricted by other terms in the case of the differential equation (2) (at least for moderate $R$ ), but a precise cancellation may not occur at some intermediate step in the numerical solution of the same equation. In order to avoid this potential problem, 
the term $q_{3} w$ was frozen at its level from the previous ( $n$ th) iteration if $q_{3}>0$, thus yielding $(14 a, b)$. The numerical scheme represented by (14) proved stable ( $R$ up to $O(200)$ was achieved in the application to buoyancy-driven motion of a bubble that is reported in Part 2, for example) and accurate (when compared with exact solutions such as the Hill's spherical vortex).

It is well known that ADI schemes can be accelerated substantially by using a sequence of iteration parameters ('timesteps'). We did a number of experiments with 'frozen ' boundary conditions and a sequence of five parameters, distributed (following Wachspress; see e.g. Ames 1969) between their minimum and maximum values in such a way as to form a geometric progression. The minimum and maximum parameters are, in principle, determined by the bounds of eigenvalues of the difference operator, but in practice can be found experimentally to yield the best convergence.

The numerical experiments with 'frozen' boundary conditions were very encouraging. For example, the complete convergence for the covariant Laplace equation with $f=\pi \xi$ (similar to the Laplace equation in polar coordinates) was obtained in only three cycles, i.e. 15 iterations, starting from a very distant and irregular initial guess. The optimal parameters ('timesteps') found experimentally for this equation were $\tau_{\min }=0.25 \times 10^{-3}$ and $\tau_{\max }=0.22$, only slightly expanding the range given by the theoretical values for an ordinary Laplace equation on a unit square with a uniform grid size $h=0.025$, which are $\tau_{\min }=\frac{1}{2} h^{2}, \tau_{\max }=2 / \pi^{2}$.

Similarly good convergence was obtained for the vorticity equation (with frozen stream-function field and boundary conditions), though the optimal range of parameters diminished as the Reynolds number increased, so that, for example, at $R=200$ the optimal bounds of 'timesteps' were $\tau_{\min }=0.1 \times 10^{-2}, \tau_{\max }=0.25 \times 10^{-1}$, and about 35 iterations were necessary for convergence.

However, experiments with the full nonlinear problem, where the boundary conditions for mapping functions and vorticity are adjusted at each iteration as outlined in $\S 3$, showed that changing the 'timestep' from one iteration to another had a negative effect on convergence. Experimentally, the best convergence rate was achieved with a constant 'timestep', equal to the grid size $h$. Note, however, that in the rising-bubble problem at high Reynolds and Weber numbers $\left(R=O\left(10^{2}\right)\right.$, $W=O(10)$ based on the equivalent diameter) the stability of the computation was improved by dividing through the form (13) of the vorticity equation by the distortion function squared $f^{2}=\left(\pi \xi\left(1-\frac{1}{2} \cos \pi \eta\right)\right)^{2}$, and then applying ADI with large timesteps $\Delta t=O(10)$. This can be viewed, of course, as a rescaling of 'time' using different 'timesteps' in different regions of the computational domain; it is not clear, however, why this should be helpful at high $R$ and $W$, while not improving the convergence rate in other cases.

As indicated above, we used centred differencing for all derivatives. Centred differencing of the Navier-Stokes equations is known to often lead to 'wiggles' in the solution at high Reynolds numbers. Although this problem is often avoided by using upwind differencing, this leads to a loss of accuracy. We therefore used only centred differencing throughout, thus preserving second-order accuracy in the difference equations. Although 'wiggles' did appear at highest $R$ in the vorticity field for the rising-bubble problem (Part 2), they were small and confined to the far-downstream portion of the flow. Apparently, vorticity diffusion was strong enough in the region near the body (bubble) to prevent their appearance. Experiments showed that downstream wiggles could be minimized (or in some cases eliminated entirely) using a reduced grid size in the $\eta$-direction in the downstream region (which was accomplished easily by changing the distortion function; see figure 1) and 
switching to the Neumann conditions for vorticity (i.e. zero derivative) at the outflow part of the outer boundary $\xi=0$. Similar features were used successfully by Fornberg (1980), together with centred differencing, for the flow past a cylinder up to $R=300$.

It should be emphasized that high Reynolds number alone is not a sufficient condition for 'wiggles' to appear in numerical solutions of the Navier-Stokes equations; the structure of the flow is also important. In order to check the accuracy of the scheme, we had previously computed as a test case the Hill's spherical vortex (flow inside a sphere with a surface velocity which matches that for irrotational uniform flow past a sphere). Hill's vortex is a non-trivial exact rotational solution of the full Navier-Stokes equations at all Reynolds numbers, and as such presents an excellent test case. We used what are essentially spherical coordinates $(\xi, \eta, \phi)$ inside the sphere, so $f=\pi \xi$, and computed the steady flow on a $40 \times 40$ grid, starting from a very distant initial guess, with $\psi=0$ at all boundaries and a vorticity distribution at the surface fixed at the exact value given by Hill's solution. The converged solution inside the domain was Hill's vortex with very good accuracy (error $O\left(10^{-4}\right)$ ) at all Reynolds numbers attempted, even as high as 1000 , and no 'wiggles' appeared! This does not mean, of course, that the $40 \times 40$ grid is sufficient in general for $R=O\left(10^{3}\right)$; Hill's vortex does not contain a boundary layer, whose resolution would presumably require a finer grid.

\subsection{Application of the tangential-stress condition}

The condition (8) of zero tangential stress at the fluid interface is used to obtain the boundary vorticity values. In most cases, this can be done in the simple explicit manner

$$
\omega^{n+1}=\omega^{n}-\beta_{\omega}\left(\omega^{n}-2 \kappa_{(\eta)} u_{\eta}^{n}\right)
$$

pioneered by Dorodnitsyn \& Meller (1968) and Israeli (1970) for a solid wall, and extended later for the case of a fluid-fluid interface by Rivkind \& Ryskin (1976) (for details see Ryskin 1980). Here $\beta_{\omega}$ is a positive relaxation parameter whose optimal value must be found by numerical experiment; typical values are $O\left(10^{-2}\right.$ to $\left.10^{-1}\right)$.

The corresponding condition $\omega^{n+1}=\omega^{n}+\beta_{\omega} u_{\eta}^{n}$ for a solid boundary can be used effectively for any smooth boundary shape. However, in the case of a fluid-fluid boundary, the condition (15) leads to a stable computation of vorticity only for interfaces of convex shape (where $\kappa_{(\eta)}>0$ ) or slightly concave shapes. For strongly concave interface shapes, condition (15) leads to instability.

In this latter case of a concave interface, an implicit method was successfully implemented for calculation of the boundary vorticity. This method, first suggested by Ghia \& Davis (1974), is much more complicated than the explicit method based on (15). The basic idea for any implicit scheme would be to determine the relationship between the boundary values for $u_{\eta}$ and the boundary values of vorticity, i.e. $u_{\eta}(\omega)$, thus giving from (8) (with $\kappa_{(\eta)}$ known) an equation for the vorticity, i.e.

$$
\omega-2 \kappa_{(\eta)} u_{\eta}(\omega)=0 \text {. }
$$

The difficulty with this approach is to estimate $u_{\eta}(\omega)$, since $u_{\eta}$ is obtained from the stream function, which, in turn, depends on the entire vorticity field, i.e. the dependence of $u_{\eta}$ on the boundary values of $\omega$ is extremely complicated and non-local ( $u_{\eta}$ at some given boundary point depends on $\omega$ at all boundary points). Even if one were to assume heuristically that the dependence on local values of $\omega$ is most important, and seek to find this dependence, one would have to solve for new vorticity and stream-function fields with $\omega$ varied at the point of interest and 'frozen' at all other boundary points, and this would need to be done separately for every boundary 
point in turn. Such an approach is clearly out of the question owing to the extreme expenditure of computer time that would be required.

However, Ghia \& Davis (1974) suggested an idea to circumvent this difficulty. Instead of doing a full iteration on $\omega$ and $\psi$, one does a half-step in $\omega$ that is implicit along the lines normal to the boundary (i.e. along the $\xi$-lines) and then immediately a half-step in $\psi$ along the same $\xi$-lines, using the new values of $\omega$. Thus the interdependence in the direction parallel to the boundary is disconnected, and a single half-iteration on the whole field provides the necessary dependence of $u_{\eta}$ on $\omega$ at the same boundary point for all boundary points at once. Of course, an error is introduced by using half-step values of $\omega$ and $\psi$ instead of the full-step, but this error goes to zero with convergence, since the half-step and full-step fields are equal on the converged solution in ADI.

It may also be noted that the function $u_{\eta}(\omega)$ can be expressed ('locally') as a linear function of $\omega$ if the solution of the discrete equations for $\omega$ along the $\xi$-lines (which are linear) is obtained as a sum of a 'particular' solution $\omega_{\mathrm{p}}$, with $\omega_{\mathrm{P}}$ set equal to zero on the boundary, and a 'homogeneous' solution $\omega_{\mathrm{H}}$ of the same equations with the right-hand side (i.e. the terms not including $\omega$ ) set equal to zero but with a non-zero boundary value for $\omega_{\mathrm{H}}$ ( $\mathrm{say} \omega_{\mathrm{H}}=1$ ). The two resulting solutions for $\omega$ are then used separately to obtain two solutions of the discrete equations for $\psi$ along the same $\xi$-line (which are also linear), and the velocity at the boundary is then obtained as a sum of two parts - one 'particular' part due to $\omega_{\mathrm{P}}$ and one 'homogeneous' part due to $\omega_{\mathrm{H}}$. Thus, if the actual vorticity is $\omega$ at the boundary, the velocity will be $u_{\eta}=u_{\eta \mathrm{P}}+\omega u_{\eta \mathrm{H}}$, and solution of $(8)$ for $\omega$, with $u_{\eta}$ expressed as a linear function of $\omega$, is a trivial task.

The rate of convergence of our numerical scheme is about the same with either the explicit or implicit prescription of boundary vorticity. Therefore, since the implicit method takes more computer time, we used it only for strongly concave interface shapes where the explicit scheme failed.

\subsection{Use of the normal-stress balance to determine interface shape}

We have already indicated in $\$ 3$ that the steady shape of a free interface is determined in our solution scheme via an iterative process in which the imbalance between the total normal stress and capillary forces for a given estimated shape of the interface is used to obtain an 'improved' shape for the next iteration - i.e. a shape for which the imbalance is decreased (or condition (9) more nearly satisfied). Although a number of methods may be suggested for obtaining this improved estimate of boundary shape, the applications of the solution technique considered to date suggest a heuristic approach as providing the greatest degree of stability and most rapid approach to the final steady state. In effect, this approach is equivalent to considering any local excess of total normal stress to capillary forces, i.e.

$$
\Pi(\eta) \equiv \tau_{\xi \xi}-\frac{4}{W}\left(\kappa_{(\eta)}+\kappa_{(\phi)}\right)
$$

as a kind of normal 'force' causing a local displacement of the interface in the direction of the 'force', the magnitude of the local displacement being proportional to $\Pi(\eta)$.

Before going on to describe the detailed implementation of this simple heuristic method, it is perhaps worth discussing briefly two alternatives that were not chosen. The most obvious would be to treat the normal-stress boundary condition (9) as a non-homogeneous differential equation for the boundary-shape functions 
$x(1, \eta), \sigma(1, \eta)$ with the total stress $\tau_{\xi \xi}$ calculated from the stream-function and vorticity fields corresponding to the interface shape at the previous iteration. However, this technique, utilized in an explicit manner as described above, is not suitable for large values of $W$. This is because it assumes implicitly that the shape is determined by a balance between the total stress $\tau_{\xi \xi}$ (including hydrostatic and dynamic pressure plus viscous stress contributions) and capillary forces, whereas for large $W$ the capillary forces are negligible (except possibly in regions of very high curvature), and the interface shape is actually determined by the condition that the viscous stress and the hydrostatic and dynamic pressure distributions balance in such a way that $\left.\tau_{\xi \xi}\right|_{\xi=1} \approx 0$. Another possible method of calculating the steady interface shape is to treat the whole problem in a genuine transient mode, thus applying the two stress relationships (8) and (9) as boundary conditions to determine the instantaneous stream-function and vorticity fields, and using the kinematic condition (i.e. the transient generalization of (7)) to increment the interface position based on the magnitude and sign of the normal velocity at each point. Although this approach appears viable to us, and would, in fact, be necessary to obtain solutions in a transient problem, the means of using (9) as a boundary condition for determination of the stream-function and vorticity fields is not obvious, and may yield a quite unstable solution algorithm since it involves derivatives of $\psi$ and $\omega$ rather than the values themselves. It must be admitted, however, that we have not so far devoted much effort to this approach, and it may ultimately prove satisfactory for both transient and steady-state calculations.

The previous efforts to compute free-boundary solutions that we are aware of utilized finite-element techniques (cf. Silliman \& Scriven 1980; Saito \& Scriven 1981). Silliman \& Scriven tried both the normal-stress and kinematic conditions in the ways just described in the preceding paragraph. As expected, they found that the normal-stress iteration diverged when a parameter, characterizing the relative magnitude of capillary pressure and stress, was below $O(1)$. The kinematic iteration diverged when the same parameter was above $O(1)$. Eventually they abandoned both of these approaches and switched to a global Newton method (Saito \& Scriven 1981), with the free-boundary shape included in the system of equations and solved simultaneously with the dynamics. Note that it is quite easy to include the boundary shape in the Newton method of Saito \& Scriven (1981), since their finite-element grid is essentially a mapping of the deforming physical domain onto a rectangle in the computational domain using an explicit algebraic formula (with a consequence, incidentally, that the resulting grid is non-orthogonal (see Ettouney \& Brown 1983)). The Newton process has great advantages (quadratic convergence, generality, availability of the Jacobian matrix which can be used to trace non-unique solutions, etc.). But it also has some severe drawbacks, including the necessity to invert a huge Jacobian matrix (extremely time-consuming). Fornberg (1980) required 1-2 min of computer time for a single case on the CDC STAR-100 computer which is $O\left(10^{3}\right)$ faster than our VAX-11/780, on which our algorithm takes from 15-60 min for a single case (for comparable grids).

Let us now consider the details of our current technique, which is heuristic in concept, but is extremely simple and exhibits rapid and stable convergence to the final steady-state interface shapes. As noted before, we modify the interface shape at each step in the overall iteration scheme by slightly moving points of the surface in the normal direction by an amount proportional to $\Pi(\eta)$ at the point. As explained in $I$, this cannot be done by prescribing new positions for the interface nodal points directly (and still maintain coordinate orthogonality), but must rather be done 
indirectly by changing the mapping itself via increments in the scale factor $h_{\xi}$ at the boundary $\xi=1$. We have shown in I that the new $h_{\xi}$ can be expressed in terms of equivalent Neumann boundary conditions for the mapping functions $x(\xi, \eta)$ and $\sigma(\xi, \eta)$. Thus

$$
h_{\xi}^{n+1}=h_{\xi}^{n}+\beta_{h} \Pi^{n}
$$

where the new values of $h_{\xi}$ are then used to calculate updated values of $\partial x / \partial \xi$ and $\partial \sigma / \partial \xi$ at $\xi=1$, which provide the necessary boundary conditions for $x(\xi, \eta)$ and $\sigma(\xi, \eta)$. The constant coefficient $\beta_{h}$ has to be determined by numerical experiment, its typical values being $O\left(10^{-3}\right.$ to $\left.10^{-2}\right)$. Higher values of $\beta_{h}$ can be used if $\Pi(\eta)$ is 'smoothed out', e.g. by reduction of higher harmonics in its Fourier expansion.

In many applications, including the two problems discussed in Parts 2 and 3, the interface completely encloses one of the two fluids - i.e. we then have a drop or bubble in a viscous fluid. In such circumstances, the local incrementation in the location of boundary points must be done in such a way as to preserve the bubble/drop volume. Now, to first order, the change in volume between the $n$th and $(n+1)$ th iterations is

$$
\int_{0}^{1}\left(h_{\xi}^{n+1}-h_{\xi}^{n}\right) \sigma h_{\eta} \mathrm{d} \eta
$$

where the integral is taken along the boundary $\xi=1$. Since

$$
\begin{aligned}
& h_{\xi}^{n+1}-h_{\xi}^{n} \sim \Pi^{n} \\
& \int_{0}^{1} \Pi \sigma h_{\eta} \mathrm{d} \eta=0
\end{aligned}
$$

it follows that

This provides a constraint on $\tau_{\xi \xi}$, which contains a free integration constant that can be chosen at each $n$ so the constraint (19) is satisfied.

Even after this constraint has been satisfied, however, the bubble or drop may still change volume slightly at each iteration owing to higher-order effects (neglected terms in (17) and (18)) and numerical error. If left unchecked, these small changes may accumulate and eventually result in a gross error. To prevent this, a simple scaling of the whole mapping can be incorporated directly into the formula for $h_{\xi}$, i.e.

$$
h_{\xi}^{n+1}=\left(\frac{4}{V^{n}} \pi\right)^{\frac{1}{3}} h_{\xi}^{n}+\beta_{h} \Pi^{n}
$$

where $V^{n}$ is the volume of the bubble at the $n$th iteration, i.e.

$$
V=\left|\pi \int_{0}^{1}\left(\sigma^{2} \frac{\partial x}{\partial \eta}\right)_{\xi=1} \mathrm{~d} \eta\right|
$$

\section{Application to the problems of bubble motion}

The technique described above has been applied to two problems of bubble motion: the rise of a bubble through an unbounded, quiescent fluid, and a bubble in uniaxial extensional flow. The physical results of these solutions will be discussed in Parts 2 and 3 . Here we will touch only upon the numerical aspects of implementing the scheme described above.

The far-field boundary conditions for these two problems are

1. the rising bubble: $\psi \sim \frac{1}{2} \sigma^{2}, \quad \omega \rightarrow 0$ for $\xi \rightarrow 0$;

2. the bubble in an extensional flow: $\psi \sim \frac{1}{2} x \sigma^{2}, \omega \rightarrow 0$ for $\xi \rightarrow 0$. 


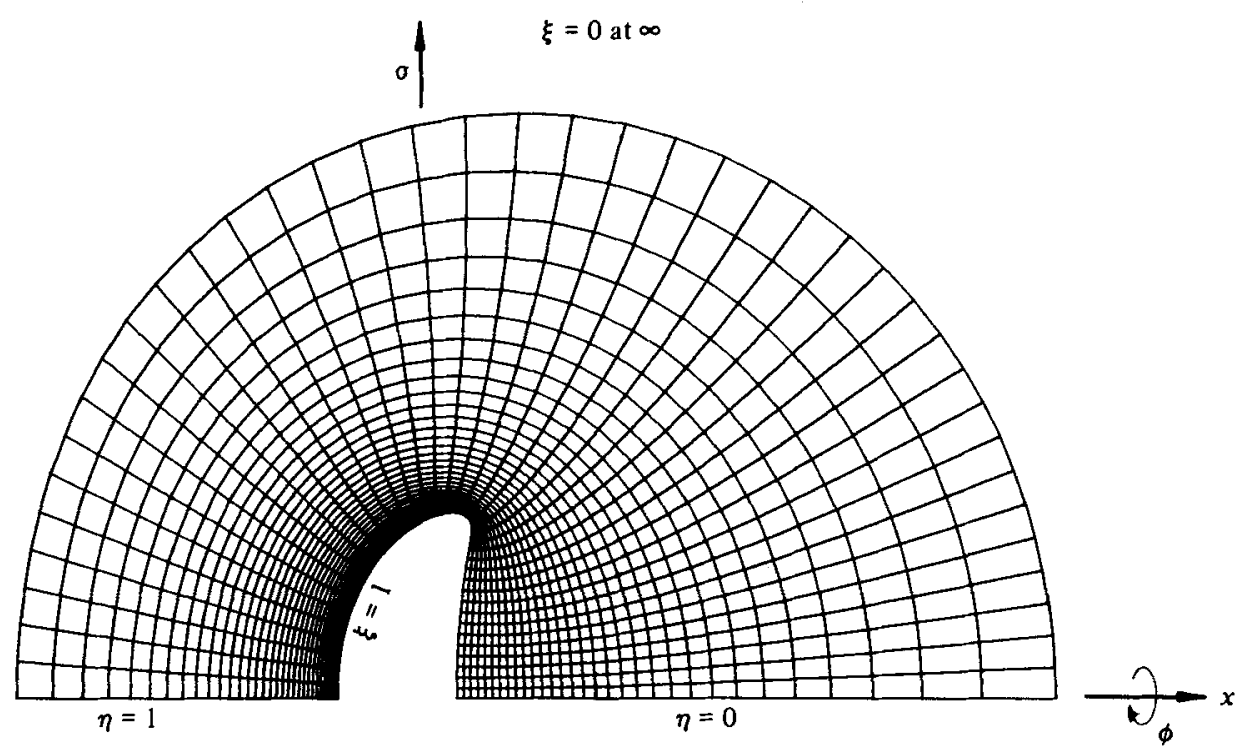

Figure 1. The boundary-fitted coordinate system for a rising bubble.

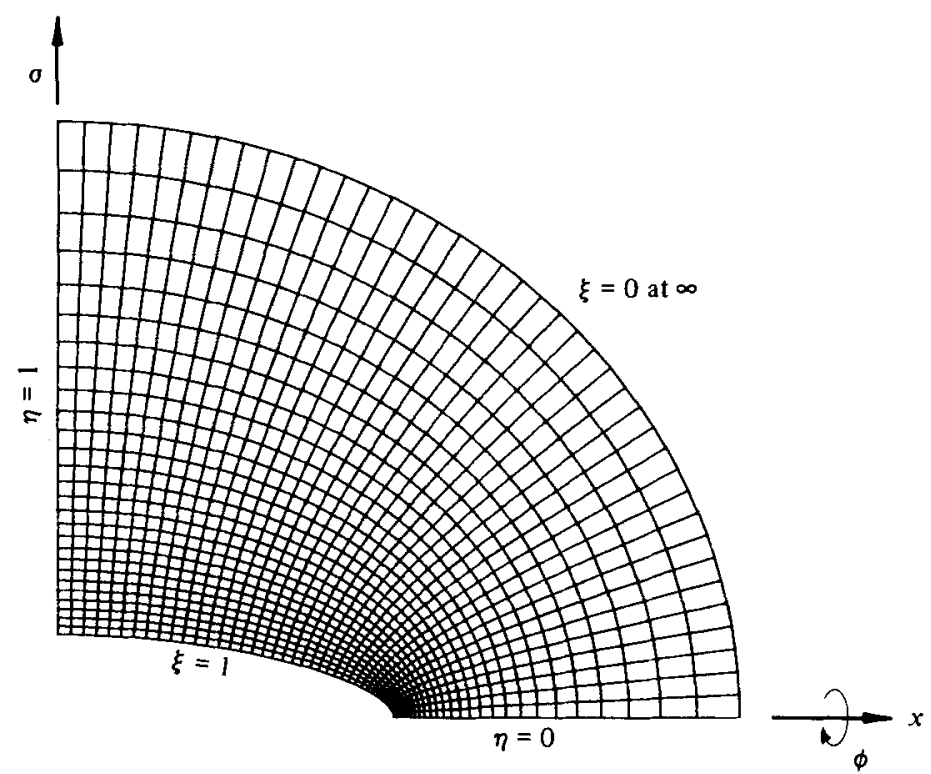

Figure 2. The boundary-fitted coordinate system for a bubble in extensional flow.

The second problem possesses, in addition to axial symmetry, symmetry with respect to the equatorial plane; therefore only the $x \geqslant 0, \sigma \geqslant 0$ quadrant needs to be considered.

The distortion function $f$ was chosen as

1. the rising bubble: $f=\pi \xi\left(1-\frac{1}{2} \cos \pi \eta\right)$

(this gives a denser grid downstream of the bubble, where it is desirable in order to give better resolution of the wake structure and also to prevent 'wiggles' in the solutions at high $R$ );

2. bubble in extensional flow: $f=\pi \xi$.

In figures 1 and 2, examples of the coordinate systems generated for these two 
problems are given. Only parts of the systems are drawn; each coordinate system actually extends to infinity. Of course, infinite numbers cannot be handled by a computer, and the most common approach of earlier investigators for approximating solutions of unbounded-domain problems has been simply to truncate the computational grid at some finite distance. We use a different approach in which a mapping is constructed from the unit square $0 \leqslant \xi, \eta \leqslant 1$ to the full infinite domain in physical space by a two-step procedure: first an orthogonal mapping is computed numerically, which maps the $(\xi, \eta)$ unit square to an auxiliary finite domain in $\left(x^{*}, \sigma^{*}\right)$-coordinates $\left(x^{*}, \sigma^{*}\right.$ satisfy (1)); then a conformal inversion $x+\mathrm{i} \sigma=\left(x^{*}-\mathrm{i} \sigma^{*}\right)^{-1}$ is used to map the $\left(x^{*}, \sigma^{*}\right)$ auxiliary domain to the infinite domain in the $(x, \sigma)$ physical space. The composite mapping $x(\xi, \eta), \sigma(\xi, \eta)$ is orthogonal; its scale factors are simply connected with the scale factors of the auxiliary mapping, computed numerically (see I). Therefore the derivatives of scale factors and $\sigma$, which appear in (2) and (3), can also be obtained at all interior points (i.e. excluding $\xi=0$ ) if the derivatives of their counterparts in the auxiliary mapping are computed, and these can be evaluated easily since all quantities of the auxiliary mapping are finite. Finite-difference equations are written only at interior points, and therefore all coefficients in the equations will be finite even though the $(x, \sigma)$-domain will be infinite.

What remains then is to make sure that the unknowns and boundary conditions are also finite. The vorticity presents no problem, but the stream function tends to $\infty$ as $\xi \rightarrow 0$. Therefore a new unknown stream function $\psi^{*}$ was introduced as follows:

1. the rising bubble: $\psi^{*} \equiv \psi-\frac{1}{2} \sigma^{2}\left(1-\xi^{3}\right)$;

2. the bubble in an extensional flow: $\psi^{*} \equiv \psi-\frac{1}{2} x \sigma^{2}\left(1-\xi^{5}\right)$.

The subtracted terms represent the irrotational-flow solutions of the respective problems for a spherical bubble, but have no simple interpretation when the bubble shape is non-spherical. The above operation removes the singularity that occurs in $\psi$ at $\xi=0$, at the same time preserving the homogeneous boundary conditions on $\psi$ at the other three boundaries.

The boundedness of $\psi^{*}$ at $\xi=0$ is a sufficient condition for solution, since $\xi=0$ is a singular point of the differential equation (see e.g. Morse \& Feshbach 1953, p. 713); and we expect $\psi^{*}$ to be bounded on the basis of Oseen-type solutions. Numerically, any $O(1)$ number (say 0 ) can be used as a boundary condition for $\psi^{*}$ at $\xi=0$, with the particular choice influencing the solution by no more than the order of accuracy of the numerical scheme.

Finally, it should be noted that a minor change is required in condition (16) owing to the fact that the final mapping is obtained from the auxiliary mapping $x^{*}(\xi, \eta)$, $\sigma^{*}(\xi, \eta)$ by the inversion described above. This means that a point on the boundary $\xi=1$ of the auxiliary mapping should be moved inward if the corresponding point of the final mapping is to move outward corresponding to a positive value of $\Pi(\eta) \equiv \tau_{\xi \xi}-(4 / w)\left(\kappa_{(\eta)}+\kappa_{(\phi)}\right)$. Thus condition (16) must be modified to the form

$$
h_{\xi}^{*(n+1)}=h_{\xi}^{*(n)}-\beta_{h}\left(r^{*}\right)^{2} \Pi^{(n)} \text { at } \xi=1 \text {, }
$$

where the factor $\left(r^{*}\right)^{2}=\left(x^{*}\right)^{2}+\left(\sigma^{*}\right)^{2}$ is inserted because the scale factors for the auxiliary and final mappings are related according to $h_{\xi}=h_{\xi}^{*} /\left(r^{*}\right)^{2}$ (see I).

The overall scheme for solution of the four elliptic equations $((2),(3)$, and the two equations for the mapping functions $x^{*}(\xi, \eta)$ and $\left.\sigma^{*}(\xi, \eta)\right)$ subject to the boundary conditions indicated earlier for $\psi^{*}, \omega$ and in I for the mapping functions, has already been described. In the present case, all of the basic computations were performed on a uniform $40 \times 40$ grid in the $(\xi, \eta)$-domain $0 \leqslant \xi, \eta \leqslant 1$. A number of checks were made to assess the accuracy of the resulting solutions. One was to insure that the 
final bubble shapes were consistent with the geometric constraint that is known as the Gauss-Bonnet theorem (cf. Sokolnikoff 1964). This theorem states that the integral of the total (Gaussian) curvature $K$ over a smooth surface, topologically equivalent to a sphere, should equal $4 \pi$. Since the normal curvatures $\kappa_{(\eta)}$ and $\kappa_{(\phi)}$ are also the principal curvatures (Dupin's theorem, see Sokolnikoff 1964, p. 195), we have $K=\kappa_{(\eta)} \kappa_{(\phi)}$, and the integral is computed easily. The final bubble shapes always satisfied the Gauss-Bonnet theorem to within $1 \%$.

A second check was more physical, and involved comparisons between the drag coefficients of the rising bubble, computed respectively on the basis of the total rate of energy dissipation via the Bobyleff-Forsythe formula (see Ryskin 1980) and by integration of the pressure and viscous stresses over the bubble surface. It may be anticipated that the latter values, dependent only on surface quantities, should be the more accurate of the two, and the surface-force integration was adopted as our basic method. The drag coefficients calculated by the two methods were found to be in good agreement in most cases (see table 1). The level of agreement was actually quite surprising, since the dissipation calculation involves integrating the squared vorticity over the whole solution domain - an operation that magnifies the effects of errors in the vorticity at large distances away from the bubble. However, at the largest values of $R$ and $W$ the differences were considerably larger. We believe that these differences in the calculated drag coefficients provide a conservative view of the accuracy of our numerical solutions. As we have already noted, the dissipation calculation weighs heavily the vorticity values at a large distance from the bubble surface, and this is just the point where one might expect the largest errors to appear. Indeed, for the highest $R$ and $W$ considered, some evidence of 'numerical noise' was present in the vorticity fields far from the bubble and recirculating wake, indicating that we are approaching the limits of usefulness of the $40 \times 40$ mesh. It should be noted, however, that relatively small errors in the vorticity at large distances from the bubble $\left(\leqslant 2 \%\right.$ of $\omega_{\max }$ ), especially downstream and outside the wake, would not be expected to influence the solution much in the vicinity of the bubble surface. Support for this point of view may be found in the recent work of Fornberg (1980), who showed that the major effect of downstream boundary conditions on the accuracy of numerical solutions for streaming flow past a cylinder was in the stream function, with the precise form of the vorticity boundary conditions playing a relatively minor role.

Finally, as indicated above, a comparison was made for one case of the rising-bubble problem, $R=20$ and $W=12$ (a highly deformed bubble), with a solution obtained on a finer $80 \times 80$ grid. The computations on the $40 \times 40$ grid took approximately 15-60 min of CPU time on a VAX-11/780 for each case, starting with the solution at the same $R$ and the next-lower value of $W$ as an initial condition. The solution on an $80 \times 80$ grid required more than an order-of-magnitude increase in computer time, and therefore was computed only for a single case. As can be seen from figure 3 , the only noticeable difference in the $40 \times 40$ and $80 \times 80$ solutions in this particular case is in the intensity of motion inside the recirculating wake (whose shape is virtually identical). Since the velocities in this region are extremely small anyway (the magnitude of $\psi$ at the innermost streamline is only 0.01 ), we judge the absolute error to be acceptably small. The value of the drag coefficient is about $3 \%$ lower on the finer grid, and this probably should be used to suggest that all of the drag coefficients from the $40 \times 40$ mesh system are going to be a little above the true values.

The ultimate check of the numerical technique (and problem formulation) is, of course, a comparison with available experimental results, and also with theoretical 


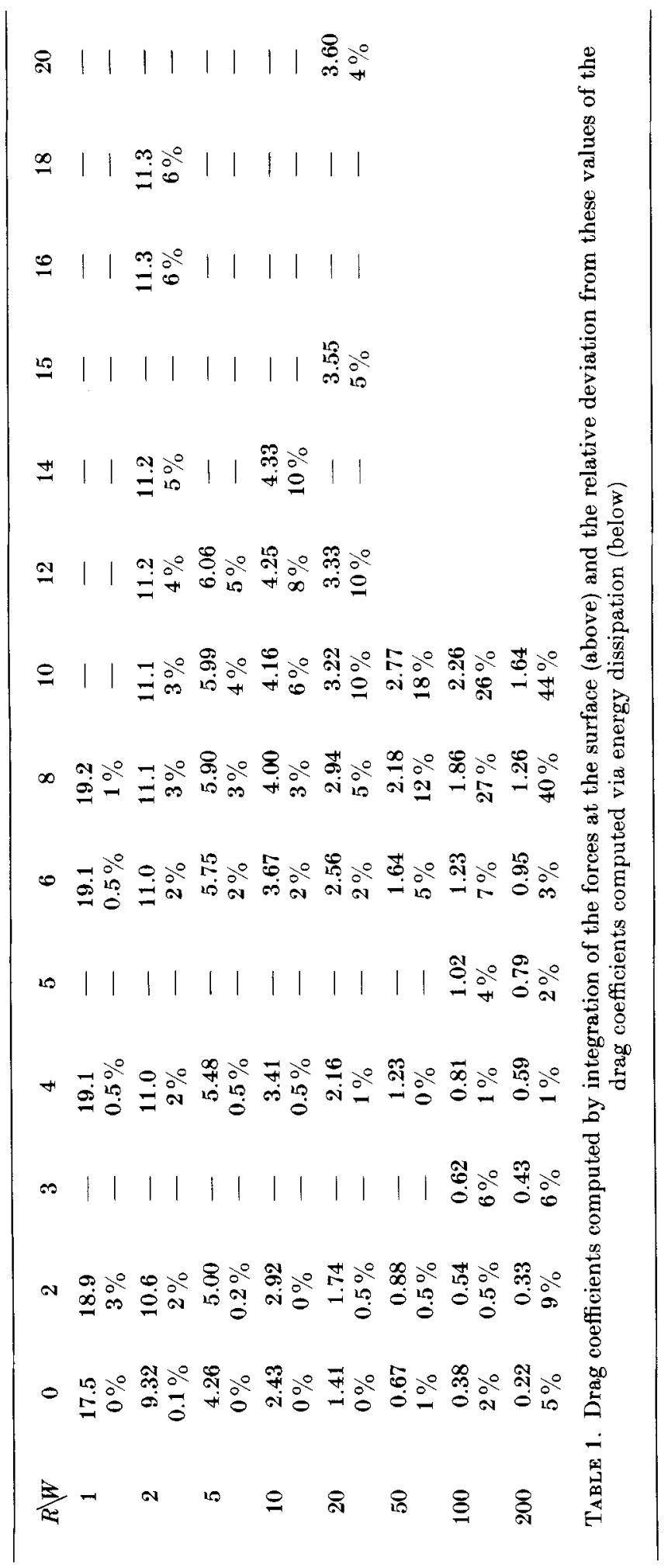



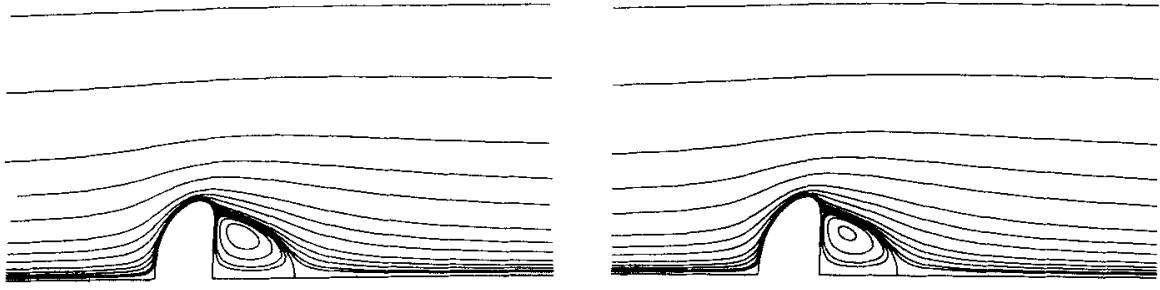

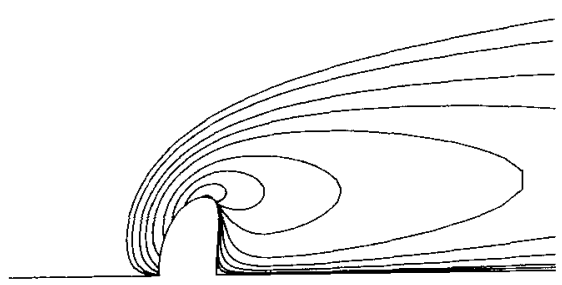

$40 \times 40$

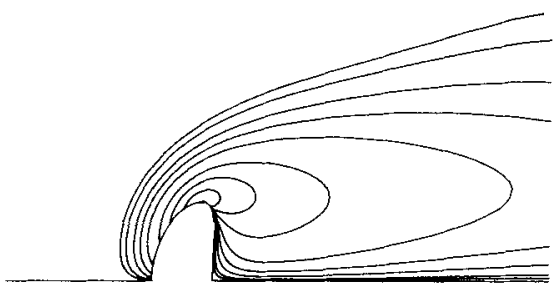

$80 \times 80$

Figure 3. Comparison of the solution for the rising bubble for $R=20, W=12$ with the $40 \times 40$ grid (left) and the finer $80 \times 80$ grid $(r i g h t)$. The contour values for the stream function are 0 , \pm 0.001 , $\pm 0.002, \pm 0.005, \pm 0.01$, etc.; for vorticity $0, \pm 0.01, \pm 0.02, \pm 0.05, \pm 0.1$, etc.

results obtained by different methods. These comparisons (which generally testify in favour of the present technique) are discussed in Parts 2 and 3, which are devoted exclusively to the physical implications of the results of our numerical solutions.

This work was supported by a grant from the Fluid Mechanics Program of the National Science Foundation.

\section{REFERENCES}

Acrivos, A. \& Lo, T. S. 1978 Deformation and breakup of a single slender drop in an extensional flow. J. Fluid Mech. 86, 641-672.

Ames, W. F. 1969 Numerical Methods for Partial Differential Equations. Barnes \& Noble.

Dorodnitsyn, A. A. \& Meller, N. A. 1968 Approaches to the solution of stationary Navier-Stokes equations. USSR Comp. Math. \& Math. Phys. 8, 205-217.

Etrouney, H. M. \& Brown, R. A. 1983 Finite-element methods for steady solidification problems. J. Comp. Phys. 49, 118-150.

Fornbero, B. 1980 A numerical study of steady viscous flow past a circular cylinder. J. Fluid Mech. 98, 819-855.

GHia, K. N. \& Davis, R. T. 1974 Corner layer flow: optimization of numerical method of solution. Comp. Fluids 2, 17-34.

IsRAELI, M. 1970 A fast implicit numerical method for time-dependent viscous flows. Stud. Appl. Maths 49, 327-349.

LeE, S. H. \& LeAl, L. G. 1982 The motion of a sphere in the presence of a deformable interface. II. A numerical study of the translation of a sphere normal to an interface. J. Colloid Interface Sci. 87, 81-106.

Miksis, M., VAnden-Broeck, J.-M. \& Keller, J. 1981 Axisymmetric bubble or drop in a uniform flow. J. Fluid Mech. 108, 89-100.

Morse, P. M. \& FeshbaCH, H. 1953 Methods of Theoretical Physics. MeGraw-Hill. 
Richtmyer, R. D. \& Morton, K. W. 1967 Difference Methods for Initial-Value Problems. Interscience.

Rivkind, V. Y. \& Ryskin, G. 1976 Flow structure in motion of a spherical drop in a fluid medium at intermediate Reynolds numbers. Fluid Dyn. 11, 5-12.

Ryskin, G. 1980 The extensional viscosity of a dilute suspension of spherical particles at intermediate microscale Reynolds numbers. J. Fluid Mech. 99, 513-529.

Ryskin, G. \& Leal, L. G. 1983 Orthogonal mapping. J. Comp. Phys. 50, 71-100.

RYSKIN, G. \& LEAL, L. G. 1984a Numerical solution of free-boundary problems in fluid mechanics. Part 2. Buoyancy-driven motion of a gas bubble through a quiescent liquid. J. Fluid Mech. 148, 19-35.

Ryskin, G. \& LEAL, L. G. $1984 b$ Numerical solution of free-boundary problems in fluid mechanics. Part 3. Bubble deformation in an axisymmetric straining flow. J. Fluid Mech. 148, 37-43.

SaIto, H. \& Scriven, L. E. 1981 Study of coating flow by the finite element method. J. Comp. Phys. 42, 53-76.

Silliman, W. J. \& Scriven, L. E. 1980 Separating flow near a static contact line: slip at a wall and shape of a free surface. J. Comp. Phys. 34, 287-313.

Sokolnikoff, I. 1964 Tensor Analysis. Wiley.

TAYLOR, G. I. 1934 The formation of emulsions in definable fields of flow. Proc. R. Soc. Lond. A $146,501-523$.

TAYLOR, G. I. 1964 Conical free surfaces and fluid interfaces. In Proc. 11th Intl Congr. Appl. Mech., Munich (ed. H. Görtler), pp. 790-796.

TAYLOR, T. D. \& Acrivos, A. 1964 On the deformation and drag of a falling viscous drop at low Reynolds number. J. Fluid Mech. 18, 466-476.

Youngren, G. K. \& ACRIvos, A. 1976 On the shape of a gas bubble in a viscous extensional flow. J. Fluid Mech. 76, 433-442. 